\title{
Receptor compaction and GTPase movements drive cotranslational protein translocation
}

Jae Ho Lee ${ }^{1}$, SangYoon Chung ${ }^{2}$, Yu-Hsien Hwang Fu' ${ }^{1, \mathrm{a}}$, Ruilin Qian ${ }^{1, \mathrm{~b}}$, Xuemeng Sun ${ }^{1, \mathrm{c}}$, Shimon

Weiss $^{2,3}$, Shu-ou Shan ${ }^{1 *}$

${ }^{1}$ Division of Chemistry and Chemical Engineering, California Institute of Technology, Pasadena,

CA 91125

${ }^{2}$ Department of Chemistry and Biochemistry, University of California Los Angeles, Los

Angeles, CA 90095

${ }^{3}$ Department of Physics, Institute of Nanotechnology and Advanced Materials, Bar-Ilan

University, Ramat-Gan, 52900, Israel

${ }^{a}$ Current address: Department of Biochemistry, Stanford University, Stanford, CA, 94305

${ }^{\mathrm{b} C u r r e n t}$ address: Department of Chemistry, University of Science and Technology of China,

Hefei, 230026, China

${ }^{\mathrm{c} C}$ Current address: Department of Chemistry, Princeton University, Princeton, NJ, 08544

*corresponding author. Email: sshan@caltech.edu

Keywords: Protein Targeting, Signal Recognition Particle, Single Molecule Spectroscopy, GTPase,

Ribosome, Protein Dynamics

Short Title: SRP receptor compaction drives protein targeting 


\begin{abstract}
Signal recognition particle (SRP) is a universally conserved targeting machine that couples the synthesis of $\sim 30 \%$ of the proteome to their proper membrane localization ${ }^{1,2}$. In eukaryotic cells, SRP recognizes translating ribosomes bearing hydrophobic signal sequences and, through interaction with SRP receptor (SR), delivers them to the Sec61p translocase on the endoplasmic reticulum (ER) membrane ${ }^{1,2}$. How SRP ensures efficient and productive initiation of protein translocation at the ER is not well understood. Here, single molecule fluorescence spectroscopy demonstrates that cargo-loaded SRP induces a global compaction of SR, driving a $>90 \AA \AA$ movement of the SRP•SR GTPase complex from the vicinity of the ribosome exit, where it initially assembles, to the distal site of SRP. These rearrangements bring translating ribosomes near the membrane, expose conserved Sec61p docking sites on the ribosome and weaken SRP's interaction with the signal sequence on the nascent polypeptide, thus priming the translating ribosome for engaging the translocation machinery. Disruption of these rearrangements severely impairs cotranslational protein translocation and is the cause of failure in an SRP54 mutant linked to severe congenital neutropenia. Our results demonstrate that multiple largescale molecular motions in the SRP•SR complex are required to drive the transition from protein targeting to translocation; these post-targeting rearrangements provide potential new points for biological regulation as well as disease intervention.
\end{abstract}


Mammalian SRP is a ribonucleoprotein complex composed of six proteins (SRP19, SRP9/14, SRP68/72, and SRP54) bound to the 7SL RNA3. The universally conserved SRP54 contains an M-domain that binds the 7SL RNA and recognizes the signal sequence or transmembrane domain (TMD) on the nascent polypeptide emerging from the ribosome exit tunnel. A special GTPase domain in SRP54, termed NG, forms a GTP-dependent complex with a homologous NG-domain in SR, and the two NG-domains reciprocally stimulate each other's GTPase activity ${ }^{4,5}$. SRP is essential ${ }^{1,6}$, and mutations in SRP54-NG cause severe congenital neutropenia with Shwachman-Diamond-like features ${ }^{7,8}$, but the molecular basis of the defect is unknown. Previous works have primarily focused on how SRP recognizes its cargo and assembles with $\mathrm{SR}^{4,5,9,10}$. These works showed that signal sequence-bearing ribosome-nascent chain complexes (RNCs) reposition SRP54-NG to dock at uL23 near the ribosome exit site, and SRP pre-organized in this 'Proximal' conformation is optimized for assembly with $\mathrm{SR}^{5,9,11}$ to deliver translating ribosomes to the ER (Fig. 1a, left and Fig. 1b).

However, multiple challenges remain for initiation of protein translocation after assembly of the targeting complex. First, SRP and the Sec61p translocase share extensively overlapping binding sites at the ribosome exit site and on the nascent polypeptide ${ }^{12,13}$. Given this overlap, how RNCs are transferred from SRP to Sec61p is a long-standing puzzle. Second, eukaryotic SR is an $\alpha / \beta$ heterodimer anchored at the ER via association of the $\operatorname{SR} \alpha$ X-domain with $\operatorname{SR} \beta$, an integral membrane protein ${ }^{14,15}$. A 200 amino acid disordered linker separates the SR NGdomain from its membrane proximal $X / \beta$ domains ${ }^{16,17}$, potentially posing another barrier for cargo loading onto the membrane-embedded Sec61p. Finally, activated GTP hydrolysis in the $\mathrm{SRP} \cdot \mathrm{SR}$ complex acts as a double-edged sword: while GTP hydrolysis drives disassembly of SRP from SR for their turnover ${ }^{18,19}$ and is not required for protein translocation $p e r s e^{18,20}$, 
premature GTP hydrolysis could abort targeting before the RNC engages Sec61p and/or other translocases ${ }^{21,22}$. Whether and how the timing of GTP hydrolysis is regulated in the mammalian SRP pathway is unclear.

Early cryo-electron microscopy (EM) analyses observed a loss of density for SRP54-NG at the ribosome exit site upon SR addition ${ }^{11,23}$. A recent cryo-EM structure of the RNC $\cdot S R P \cdot S R$ complex showed that SRP can adopt a distinct conformation in which its NG-domain, bound to SR, docks at a 'Distal' site at the opposite end of 7SL RNA where SRP68/72 is located (Fig. 1a, middle and Fig. 1c) $)^{23,24}$. These observations suggest that SRP is dynamic and undergoes largescale conformational rearrangements after assembly with SR. However, these rearrangements have not been directly observed, and no information is available about their mechanism, regulation, and function.

To address these questions, we studied global conformational changes in human SRP and SR using Förster Resonance Energy Transfer (FRET). We developed three FRET pairs that monitor distinct molecular movements in the targeting complex. Detachment of SRP54-NG from the ribosome exit was detected using a donor dye (Atto550) labeled at SRP19(C64) and an acceptor dye (Atto647N) labeled at SRP54(C12) (Fig. 1a and b, Proximal Probes or SRPProx $)^{5,9}$. Docking of SRP54-NG at the distal site was detected using a donor dye (Cy3B) labeled at SRP54(C47) and Atto647N labeled near SRP68(P149) (Fig. 1a and c, Distal Probes or SRP Dist). ) Finally, we monitored the end-to-end distance of SR using Atto550 labeled at the C-terminus of SR $\alpha$ NG and Atto647N labeled at the N-terminus of SR $\beta$ (Fig. 1a and d, Compaction Probes or SR Comp$)$. We detected FRET between all three pairs of probes using a diffusion-based single molecule technique with microsecond timescale Alternating Laser Excitation ( $\mu$ s-ALEX $)^{25-27}$ (Extended Data Fig. 1). Unless otherwise specified, all measurements were made in the complete 
targeting complex in which SRP•SR is also bound to the ribosome and signal sequence and used a soluble SR complex in which the SR $\beta$ TMD, dispensable for SRP binding and cotranslational protein translocation, is removed ${ }^{28}$. Fluorescently labeled SRP and SR retain the ability to target preproteins to the ER (Extended Data Fig. 3 and Lee JH et al. ${ }^{5}$ ). We confirmed that the tested reaction conditions did not alter photo-physical properties of fluorophores, so that the observed FRET changes can be ascribed solely to conformational changes in SRP and SR (Extended Data Fig. 2).

Signal sequence- and ribosome-bound SRPProx mainly displayed a high FRET population (Fig. 1e), indicating that SRP54-NG initially docks near the ribosome exit site as previously reported $^{5}$. In contrast, $\sim 70 \%$ of SRP Prox displayed low FRET upon SR addition (Fig. 1f), indicating that interaction with SR induces SRP54-NG to move away from the ribosome exit. The opposite was observed with the distal probes: the FRET histogram of $\mathrm{SRP}_{\text {Dist }}$ was dominated by low FRET populations (Fig. 1g), whereas approximately 34\% of the complex acquired high FRET upon addition of SR (Fig. 1h), indicating acquirement of the distal state in this population. These results provide direct evidence that the SRP-SR NG-complex detaches from the ribosome exit site, where it initially assembles ${ }^{5}$, and docks at the distal site where SRP68/72 is located. Intriguingly, the population of the targeting complex that exhibited low FRET detected by the proximal probes far exceeded the high FRET population detected by the distal probes, suggesting that the targeting complex samples additional conformations in which the NG-complex is not stably docked at either the ribosome exit or the distal site.

Finally, we monitored the global conformational changes of SR using the compaction probes that report on the proximity of its folded NG- and X/ $\beta$-domains (Fig. 1a and d). As SR was implicated in ribosome binding ${ }^{16,17}$, we first measured the conformation of free SR with the $80 \mathrm{~S}$ ribosome 
present. The smFRET histogram of $\mathrm{SR}_{\text {comp }}$ exhibited a main peak at FRET $\sim 0.15$ (Fig. 1i), indicating that the NG- and $X / \beta$ domains are separated by $\geq 90 \AA$ in free SR. When signal sequence- and ribosome-bound SRP were present, however, the FRET distribution of SR Comp became broader and shifted to higher FRET with a major peak at FRET $\sim 0.7$ (Fig. 1j). These results show that SR undergoes a global compaction upon binding with cargo-loaded SRP, bringing its NG-domain much closer to the membrane-proximal X/ $\beta$-domain.

To understand the molecular mechanisms that drive these conformational changes, we introduced mutations that disrupt the interaction surfaces of SR $\alpha$ NG with SR $\beta$, SRX, or SRP68/72 (Fig. 2a, Extended Data Fig. 4a and 4b). Additionally, we characterized one of the SRP54 mutations (G226E) that cause congenital neutropenia with Shwachman-Diamond-like features ${ }^{7,8}$ (Fig. 2a and Extended Data Fig. 4a). None of the mutations impaired SRP-SR complex assembly or their reciprocal GTPase activation (Extended Data Fig. 4c-e). As efficient SRP-SR interaction requires both the ribosome and signal sequence ${ }^{5}$, these results also ruled out defects of these mutants in ribosome binding or signal sequence interaction. Thus, all of the defects observed in the following analyses are caused by conformational defects that occur after SRP-SR assembly.

ALEX measurements suggest that these mutants block the conformational rearrangements in the targeting complex at distinct steps. SRP54(G226E), which causes congenital neutropenia ${ }^{7,8}$, severely impaired all three conformational rearrangements in the targeting complex (Figs. 2b-d; summarized in Fig. 2k-m, brown). Similar albeit less pronounced defects were observed with mutants $\operatorname{SR}(\Delta 361)$ and $\operatorname{SR}(\Delta 371)$ that disrupt the intramolecular interactions between $\operatorname{SRX}$ and SR $\alpha$ NG: they not only compromised SR compaction, as expected (Extended Data Fig. 5c and 5f; summarized in Fig. 2m, orange), but also impaired the detachment of the NG-domain complex from the ribosome exit site and its docking at the distal site (Extended Data Fig. 5a, b, d and e; summarized 
in Figs. 2k and 21, orange). These results suggest that the intramolecular interactions within SR are crucial for the movement of the NG-complex from the proximal to the distal site. Reciprocally, all the mutations that disrupted distal site docking also reduced SR compaction to varying degrees (Fig. $2 \mathrm{~m}$ ), suggesting that the distal state stabilizes a highly compact SR. Nevertheless, several mutants showed more specific defects. $\operatorname{SR}(\Delta 572)$, which disrupts the contact of SR $\alpha$ NG with SRP68/72 (Figs. 2a, Extended Data Fig. 4a and b, blue), specifically destabilized distal site docking but did not affect the removal of the NG-domain complex from the ribosome exit and modestly reduced SR compaction (Fig. 2e-g; summarized in Fig. 2k-m, blue). This shows that distal docking is not required for, and probably occurs after, the other rearrangements. Finally, SR(R407A) disrupted the interaction of SR $\alpha$ NG with SR $\beta$ (Figs. 2a and Extended Data Fig. 4a, 4b, 6e, green). This mutant was impaired in both of the lateral movements of the NG-domain complex as strongly as $\operatorname{SR}(\Delta 361)$ and $\operatorname{SR}(\Delta 371)$ (Fig. $2 \mathrm{~h}$ and $2 \mathrm{i}$; summarized in Fig. $2 \mathrm{k}$ and 21 , green) but was able to undergo significant compaction similarly to $\mathrm{SR}((\Delta 572)$ (Fig. $2 \mathrm{j}$ and $2 \mathrm{~m}$, green), suggesting that SR can sample the compact conformation before the other rearrangements. The distinct mutational phenotypes (qualitatively summarized in Fig. 2a) suggest a sequential model in which SR compaction precedes and potentially drives the movement of the NG-complex from the ribosome exit to the distal site of SRP.

Analysis of the dynamics of the rearrangements supported this sequential model. We first performed burst variance analysis (BVA), which detects dynamics by comparing the standard deviation of $\mathrm{E}^{*}\left(\sigma_{\mathrm{E}^{*}}\right)$ for individual molecules to the static limit, defined by photon statistics (Supplementary Methods) $^{27,29-31}$. If multiple conformations interconvert on the sub-millisecond timescale, the observed $\sigma_{\mathrm{E}}$ would be higher than the static limit, whereas $\sigma_{\mathrm{E}^{*}}$ would lie on the static limit curve if conformational interconversions are slower compared to molecular diffusion (1-5 
milliseconds) $)^{27,29-31}$. SR $\mathrm{S}_{\text {comp }}$ displayed $\sigma_{\mathrm{E}^{*}}$ values significantly higher than the static limit (Fig. $2 \mathrm{n}$, triangles versus dashed curve). This indicates that SR samples the compact state on the submillisecond timescale and is consistent with the disordered nature of the SR linker ${ }^{16,17}$. In contrast, $\sigma_{\mathrm{E}}$ for $\mathrm{SRP}_{\text {Prox }}$ and $\mathrm{SRP}_{\text {Dist }}$ are much closer to the static limit (Extended Data Fig. 6a-c). Kinetic measurements using the $\mathrm{SRP}_{\text {Prox }}$ and $\mathrm{SRP}_{\text {Dist }}$ probes further showed that exit site detachment and distal docking occur with rate constants of $0.21 \mathrm{~s}^{-1}$ and $0.07 \mathrm{~s}^{-1}$, respectively (Fig. 2o and Extended Data Fig. 6d). Thus, SR can rapidly undergo compaction upon binding of cargo-loaded SRP, followed by detachment of the NG-complex from the ribosome exit and subsequent docking at the distal site.

Structural modeling supported SR compaction as a driver for the GTPase movements. When the compacted SRP54-NG•SR structure was superimposed on SRP54-NG bound at the ribosome exit site ${ }^{9,24}$, we found that SR compaction brings SRX close to the ribosome, potentially generating a steric clash that would destabilize the proximal state and drive detachment of the NG-complex from the ribosome exit (Extended Data Fig. 6e). This is consistent with smFRET data showing that all the SR mutations that impair this detachment disrupt interactions within the SR complex $(\Delta 361$, $\Delta 371$, and R407A). An alternative model consistent with most of the data could involve initial formation of bidentate interactions of SRP with an extended SR via both the NG-domain contacts at the ribosome exit and SRX/ $\beta$ docking at the distal site, followed by SR compaction that moves the NG-complex to the distal site. Intriguingly, SRP54(G226E), which caused the most severe defects in all three rearrangements, is located at the interface between the N- and G-domains of SRP54 (Extended Data Fig. 6, brown). In the extensively-studied bacterial SRP pathway, this interface acts as a fulcrum that undergoes cooperative adjustments in both the SRP54 and SR NG-domains upon their GTP-dependent assembly ${ }^{32-34}$. These observations suggest that the cooperative rearrangements 
within the SRP•SR NG-domain complex during its assembly are coupled to restructuring of the SR linker and thus amplified into the largescale molecular motions observed here.

We next asked how the conformational changes in SRP and SR are regulated by their biological cues including the ribosome and signal sequence. The smFRET histogram of apo-SR Comp had a broad distribution without a major peak (Fig. 3a), indicating that free SR samples multiple conformations. With either the ribosome (Fig. 1i and 3a-d, dashed red line) or SRP (Fig. 3b) present, the histogram was dominated by a peak at FRET $<0.2$, indicating that binding of the ribosome or SRP induced SR into a highly extended conformation. However, when both the ribosome and SRP are present, the histogram for $\mathrm{SR}_{\text {Comp }}$ peaked at FRET $\sim 0.7$ (Fig. 3d) and was similar to that in the presence of ribosome- and signal sequence-bound SRP (Fig. 1j). Signal sequence-bound SRP also shifted the histogram of $\mathrm{SR}_{\text {Comp }}$ to higher FRET, but less effectively than ribosome-bound SRP (Fig. 3c). Thus, the ribosome and SRP cooperatively drive the compaction of SR.

We further tested how the lateral movements of the GTPase complex are regulated. We previously showed that the histogram of free SRPProx is dominated by a medium FRET population; signal sequence drives SRP to the proximal state and thus shifts the histogram toward higher FRET, whereas the ribosome induces SRP to sample at least three conformations (Lee JH et al. ${ }^{5}$ and Extended Data Fig. 7a-c). The smFRET histograms of SRP Dist $_{\text {largely mirrored these ribosome- and }}$ signal sequence-induced changes in SRP and indicated the absence of the distal state prior to SR binding (Fig. 3e-g). Addition of SR induced the distal state at FRET $\sim 0.8$ under all tested conditions (Fig. 3h-j). Surprisingly, in the presence of ribosome, the distal state was nearly two-fold more enriched (62\%) than in the complete targeting complex with signal sequence present (Fig. 3j vs $3 i$ ). These results show that distal docking of the GTPase complex is driven by SR and favored by the ribosome, but is destabilized by the signal sequence. 
Changes in the equilibrium for distal site docking with and without signal sequence is thermodynamically coupled to the changes in signal sequence interactions before and after distal docking (Fig. 3k). Therefore, the less favorable distal docking in the presence of signal sequence $\left(K_{\text {Distal }}^{\prime}<K_{\text {Distal }}\right)$ implies that the interaction of SRP with signal sequence is destabilized upon rearrangement to the distal state $\left(K^{\prime}\right.$ Sig. Seq. $<K_{\text {Sig. Seq }}$ Fig. $\left.3 \mathrm{k}\right)$. This is consistent with the weaker EM density of the signal sequence in the distal state structure compared to the RNC •SRP complex ${ }^{9,24}$, and suggests that distal site docking provides a mechanism to facilitate the handover of the nascent polypeptide from SRP to the translocation machinery.

GTP hydrolysis in the SRP•SR complex drives their irreversible disassembly and is an important regulatory point in the bacterial SRP pathway ${ }^{35,36}$. To test how the conformational rearrangements in the SRP•SR complex regulate GTP hydrolysis, we measured the stimulated GTPase activity of the targeting complex ( $\left.k_{\text {cat }}\right)$ using mutants and conditions that bias the conformational equilibria (Extended Data Fig. 4c and 4d) ${ }^{5}$. The complete targeting complexes assembled with all the SR conformational mutants displayed higher GTPase rates $\left(k_{\text {cat }}\right)$ than the wildtype complex (Fig. 4a). The value of $k_{\text {cat }}$ negatively correlated with the fraction of SRP•SR in the distal state (Fig. 4b). The lowest GTPase rate was observed with ribosome-bound SRP•SR ${ }^{5}$, which strongly favors the distal state (Figs. $4 \mathrm{~b}$ and $3 \mathrm{j}$ ). This strongly suggests that docking at the distal site inhibits GTP hydrolysis and thus increases the lifetime of the targeting complex at the ER membrane. This is consistent with our previous observation that mutations in the SRP72 C-terminus, which is positioned near the GTPase active site in the distal state structure, hyper-activated the GTPase reaction $^{24}$. On the other hand, the targeting complex bearing mutant $\mathrm{SRP}(\mathrm{G} 226 \mathrm{E})$, in which the NGdomain complex is trapped at the ribosome exit site, hydrolyzed GTP at a rate constant of $\sim 5 \mathrm{~min}^{-1}$, providing an estimate for the GTPase rate when the targeting complex is in the Proximal state. These 
observations further suggest that hyperactive GTP hydrolysis in the targeting complex primarily occurs when the NG-complex is not docked at either the ribosome exit or the distal site. Thus, conformational rearrangements tune the timing of GTP hydrolysis, possibly providing a balance between efficient SRP turnover and substrate transfer.

Finally, we tested the role of these conformational rearrangements in SRP function using a reconstituted assay that measures co-translational translocation of a model substrate, preprolactin (pPL), to ER microsomes ${ }^{5,37}$ mediated by the SRP and SR variants (Extended Data Fig. 3a). Most of the SR conformational mutants are defective in pPL translocation (Fig. 4e, 4f, and Extended Data Fig. 8a). The largest defects were observed with $\operatorname{SR}(\Delta 361)$ and $\operatorname{SR}(\Delta 371)$, which block the rearrangements at the earliest stage (Fig. 2, Fig. 4e, and 4f, orange). SR( $\Delta 572)$, which specifically blocks distal site docking, also substantially reduced translocation efficiency (Fig. 2, Fig. 4e and 4f, blue), supporting a role of the distal state in ensuring efficient protein translocation. The only exception was SR(R407A), which did not substantially affect pPL translocation despite impairments in the lateral movements of the NG-complex; this might reflect contributions from additional factors in the cell lysate and ER microsomes during translocation measurements that were not present in smFRET measurements of the purified targeting complex. Notably, mutant SRP(G226E), which causes severe congenital neutropenia ${ }^{7,8}$, strongly impaired pPL translocation (Fig. 4d and 4f, brown). Contrary to a previous report ${ }^{7}$, we found that SRP(G226E) displays basal GTPase activity and stimulated GTPase reactions with SR as efficiently as wildtype SRP (Extended Data Fig. 4d and 4f). Using a pair of FRET probes incorporated at SRP54(C47) and the SR $\alpha$ C-terminus ${ }^{5}$, equilibrium titrations further corroborated that SRP(G226E) assembles a stable complex with SR (Fig. 4c), showing that this mutant was specifically blocked in conformational rearrangements after SRP-SR assembly (Fig. 
2b-d). These results demonstrate that the post-targeting conformational rearrangements in SRP and SR play essential roles in co-translational protein translocation and can be the point of failure in devastating pathology.

The results here demonstrate that, after an RNC•SRP•SR complex assembles at the ER, multiple largescale conformational rearrangements in the targeting complex are required to initiate protein translocation (Fig. 4g). During cargo recognition, signal sequence bearing ribosomes induce SRP into a 'Proximal' conformation in which the SRP54-NG domain docks at $\mathrm{uL} 23$ near the ribosome exit (step 1$)^{5}$. In this conformation, SRP rapidly recruits SR via interaction between their NG-domains (step 2). Cooperative rearrangements in the NG-complex upon its assembly, especially those at the N-G domain interface, are sensed by the SR linker and amplified into a global compaction of SR, likely bringing the translating ribosome near the ER membrane (step 3). This compaction also drives the detachment of the GTPase complex from the ribosome exit (step 4), exposing universal docking sites at the ribosome exit site for subsequent interaction with the Sec61p translocase. A fraction of the GTPase complex docks at the distal site (step 5). In this state, the interaction of SRP with the signal sequence is destabilized to further prime the RNC for subsequent unloading, and delayed GTP hydrolysis could generate an extended time window during which the targeting complex searches for and allows the translating ribosome to engage the appropriate translocase (step 6). These post-targeting molecular movements resolve multiple mechanistic challenges during initiation of protein translocation and are demonstrable points of failure in diseases such as congenital neutropenia, and could serve as important points for biological regulation as well as disease intervention. 
a
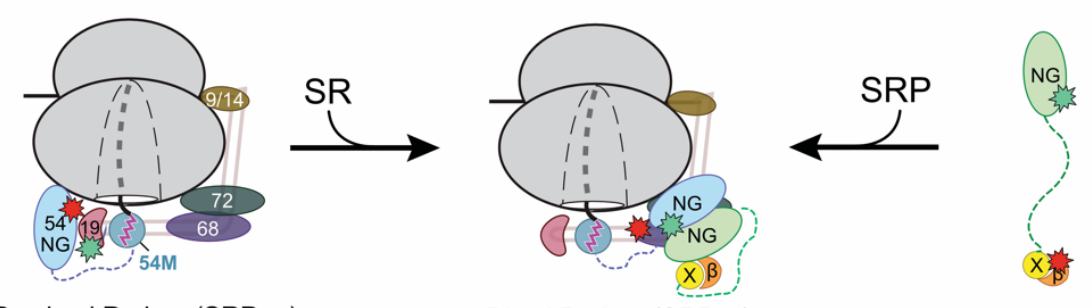

Proximal Probes $\left(\mathrm{SRP}_{\text {Prox }}\right)$

Distal Probes $\left(\mathrm{SRP}_{\text {Dist }}\right)$

b Proximal Probes

C Distal Probes
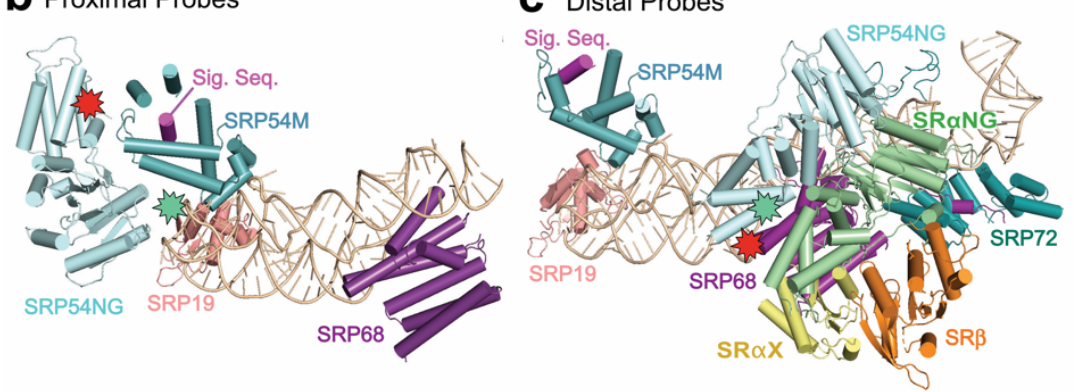

Compaction Probes $\left(\mathrm{SR}_{\text {Comp }}\right)$

d Compaction Probes

e
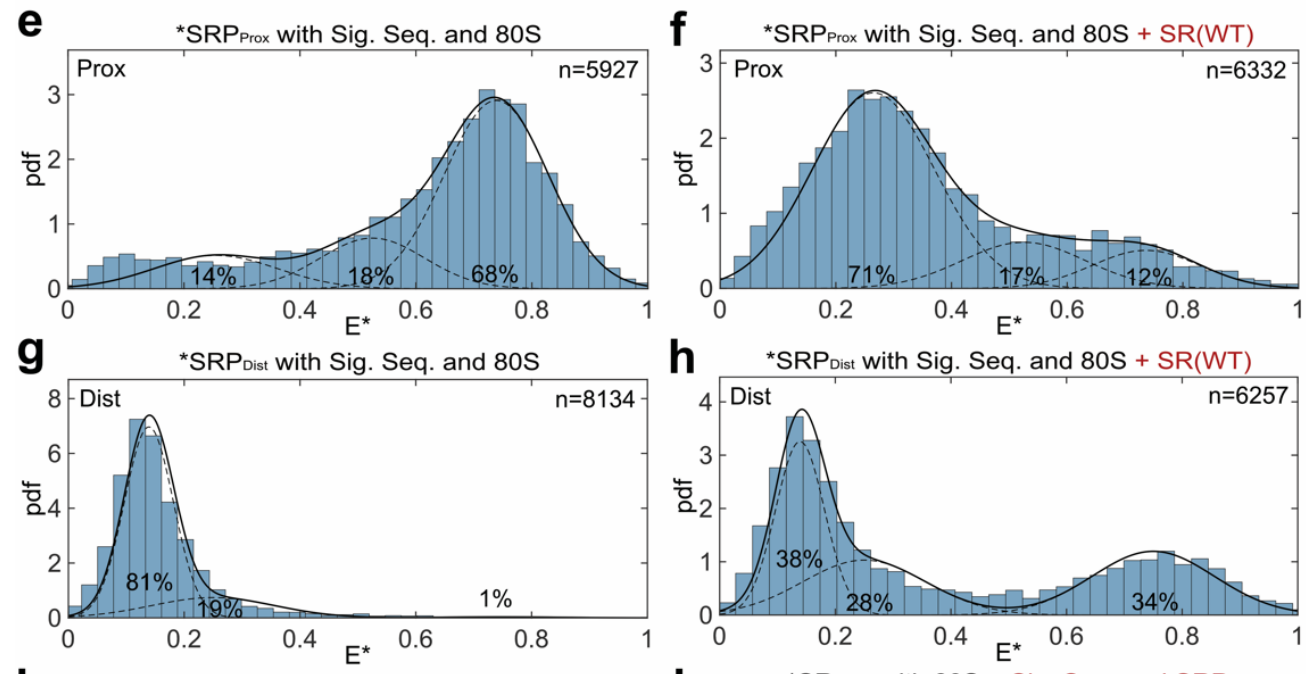

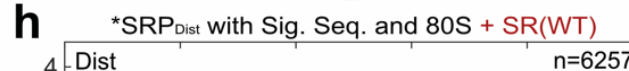
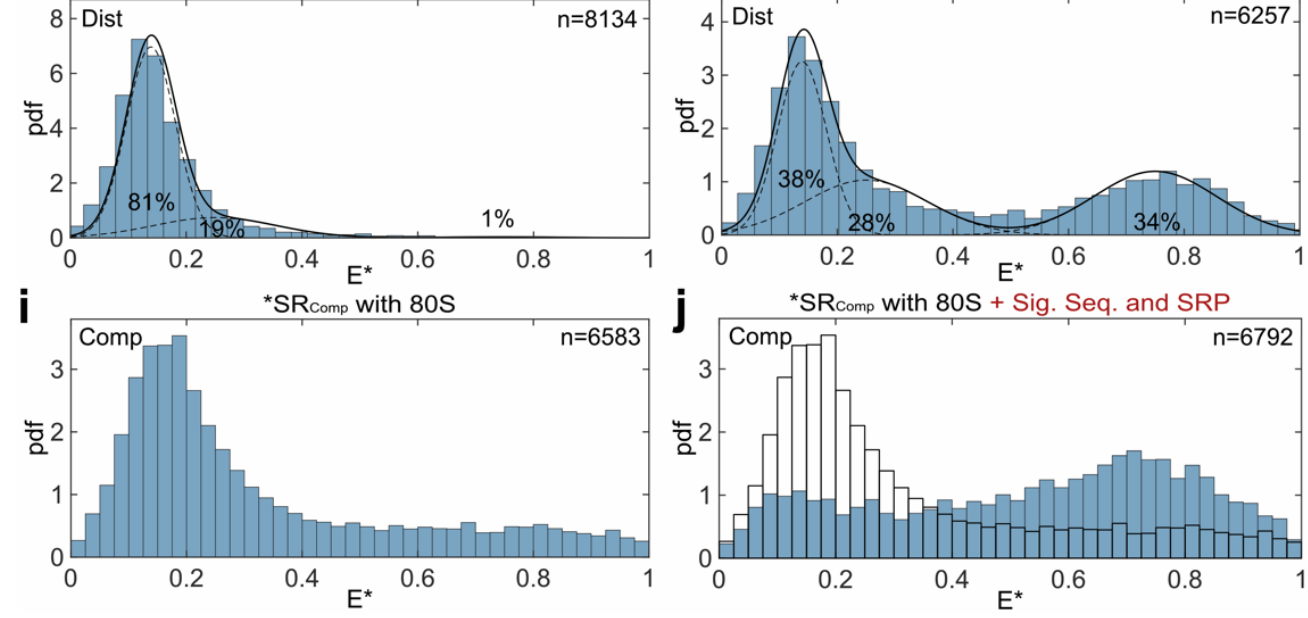

Figure 1. smFRET measurements detect multiple conformational changes upon SRP-SR assembly. a, Schematic of conformational changes in SRP and SR indicating the locations of FRET donor (green) and acceptor (red) dyes. Left, the 'Proximal' conformation of SRP in which SRP54-NG is bound near the ribosome exit site. Middle, the 'Distal' conformation in which SRP54-NG docks near SRP68/72 at the distal site. Right, the third FRET pair to measure the end-to-end distance of eukaryotic SR. b-d, Location of the FRET dyes are shown in the structures of RNC-bound SRP (b; PDB:3JAJ) and the RNC•SRP•SR complex in the distal conformation (c, d; PDB: 6FRK). The estimated distance between the dye pair is $\sim 44 \AA$ in (b), 
$\sim 39 \AA$ in (c), and $\sim 36 \AA$ in (d). e-h, smFRET histograms of signal sequence-fused SRP labeled with the Proximal $(\mathbf{e}, \mathbf{f})$ or Distal $(\mathbf{g}, \mathbf{h})$ probes without $(\mathbf{e}, \mathbf{g})$ and with $(\mathbf{f}, \mathbf{h})$ SR present. Asterisk indicates the fluorescently-labeled species. 'pdf', probability density function; E*, uncorrected FRET efficiency. ' $n$ ', the number of bursts used to construct each histogram, obtained from at least three independent measurements. The data were fit to the sum (solid line) of three Gaussian functions low, medium, and high FRET (dotted lines). The fractions of each population are indicated. $\mathbf{i}-\mathbf{j}$, smFRET histograms of SR labeled with Compaction probes in the absence (i) and presence $(\mathbf{j})$ of signal sequence-fused SRP. These histograms were not fit, because the intermediate $\mathrm{E}^{*}$ values arise from dynamic sampling of SR rather than discrete conformational states (Fig. 2n). 
bioRxiv preprint doi: https://doi.org/10.1101/2020.01.07.897827; this version posted January 8, 2020. The copyright holder for this preprint (which was not certified by peer review) is the author/funder, who has granted bioRxiv a license to display the preprint in perpetuity. It is made available under aCC-BY-NC-ND 4.0 International license.

a

\begin{tabular}{|c|c|c|c|c|}
\hline Mutation & Location & $\begin{array}{c}\text { SR } \\
\text { Compaction } \\
\end{array}$ & $\begin{array}{c}\text { Exit Site } \\
\text { Detachment }\end{array}$ & $\begin{array}{c}\text { Distal } \\
\text { Docking }\end{array}$ \\
\hline WT & NA & ++ & ++ & + \\
\hline SRP54(G226E) & SRP54 N - G interface & -- & -- & -- \\
\hline $\operatorname{SR} \propto \Delta x$ & SRX/ $\beta$ - SRaNG interface & N. D. & - & -- \\
\hline $\operatorname{SR}(\Delta 361)$ & SRX - SRaNG interface & 一 & 一 & -- \\
\hline $\operatorname{SR}(\Delta 371)$ & SRX - SRaNG interface & - & - & -- \\
\hline SR(R407A) & SR $\beta$ - SRaNG interface & + & - & -- \\
\hline $\operatorname{SR}(\Delta 572)$ & RP68/72 - SRaNG interface & + & ++ & - \\
\hline
\end{tabular}

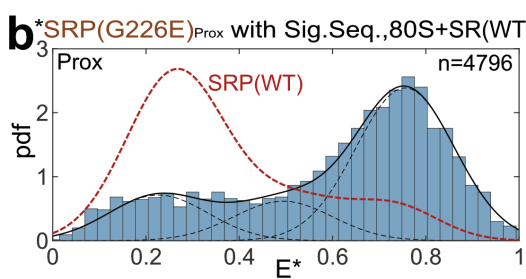

$\mathbf{e}_{3}{ }^{*}$ SRP Prox with Sig. Seq., $80 S+\operatorname{SR}(\Delta 572)$

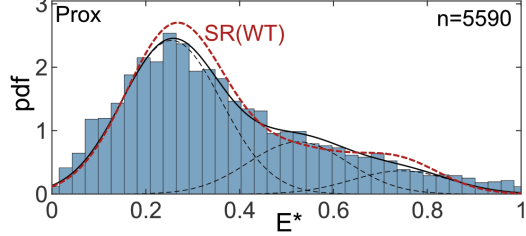

$\mathbf{h}_{3}{ }^{*}$ SRPProx with Sig.Seq., 80S +SR(R407A)

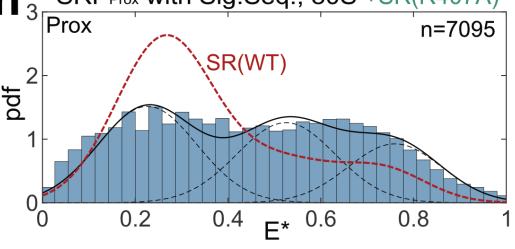

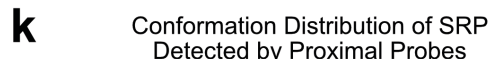
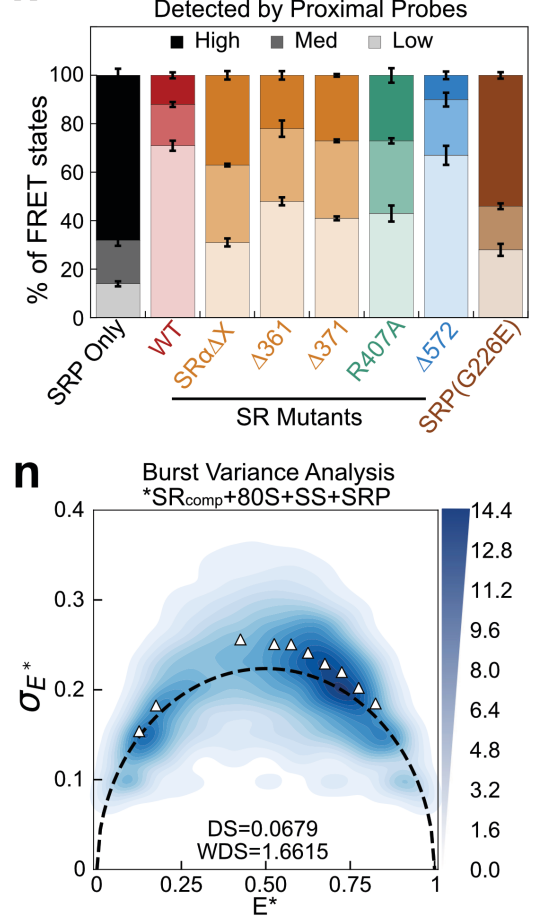

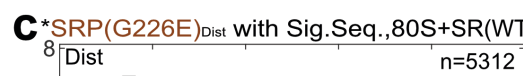

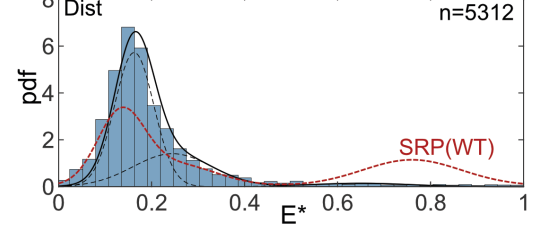

f ${ }^{*} \mathrm{SRP}_{\text {Dist }}$ with Sig. Seq., $80 \mathrm{~S}+\mathrm{SR}(\Delta 572)$

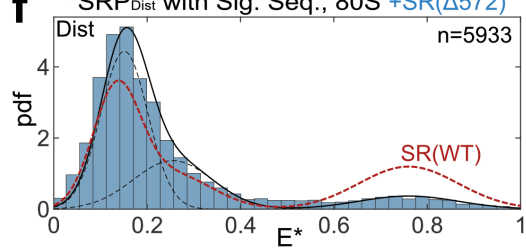

i ${ }^{*} \mathrm{SRP} \mathrm{D}_{\mathrm{Dis}}$ with Sig.Seq., $80 \mathrm{~S}+\mathrm{SR}(\mathrm{R} 407 \mathrm{~A})$

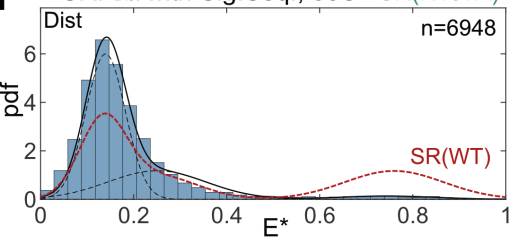

I Conformation Distribution of SRP
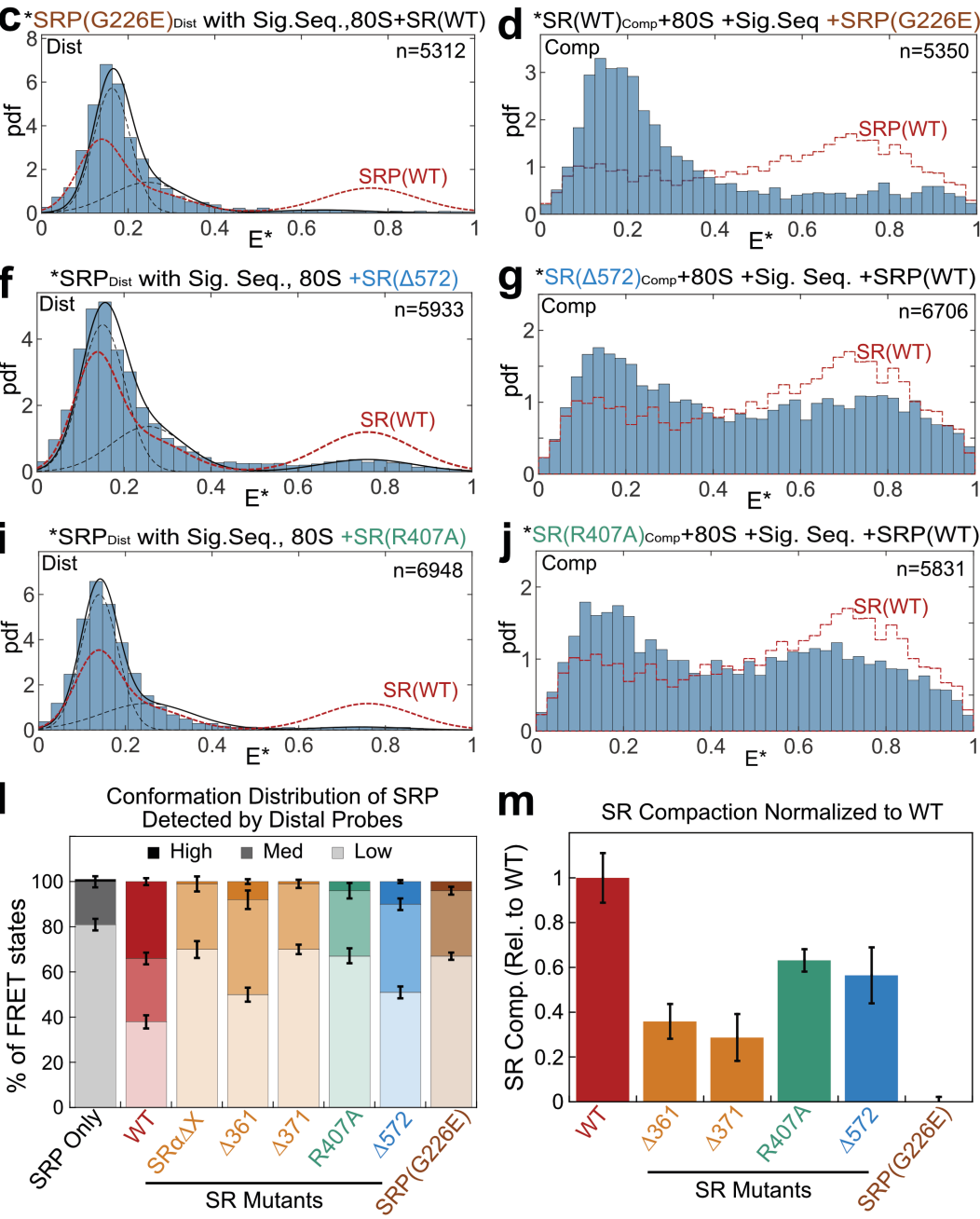

g

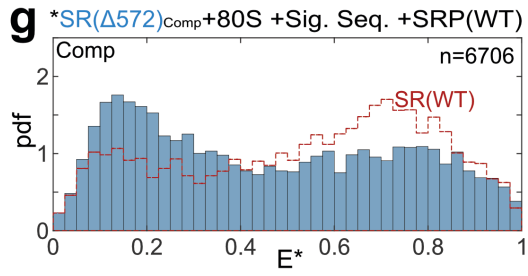

j ${ }^{*} \mathrm{SR}(\mathrm{R} 407 \mathrm{~A}) \mathrm{comp}+80 \mathrm{~S}+$ Sig. Seq. $+\mathrm{SRP}(\mathrm{WT})$

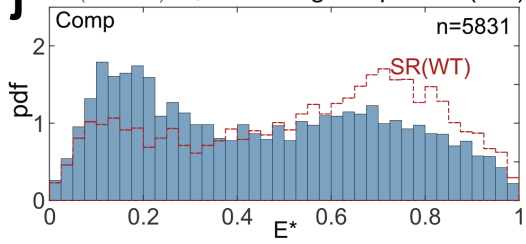

m SR Compaction Normalized to WT

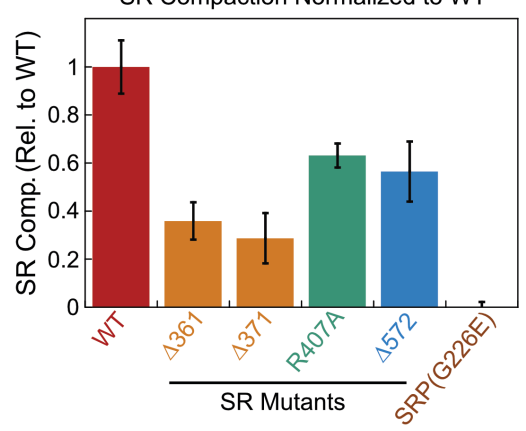

O

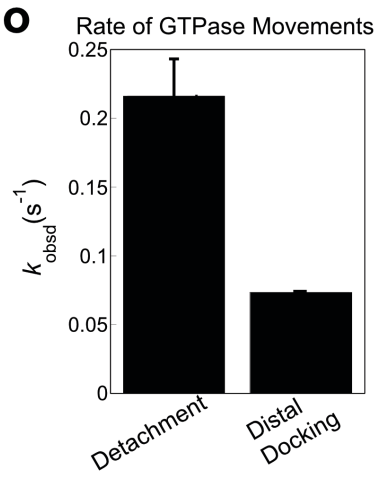


Figure 2. SR compaction drives GTPase movements in the targeting complex. a, Summary of the mutations characterized in this study and their phenotypes derived from the data in $\mathbf{b}-\mathbf{m}$. Mutations are colored based on the interactions disrupted. Details of each mutation are shown in Extended Data Fig. 4a-b. b-j, smFRET histograms of targeting complex containing mutants SRP(G226E) (b-d), SR( $\Delta 572)(\mathbf{e}-\mathbf{g})$, or SR(R407A) (h-j) detected by the Proximal (b, e, h), Distal $(\mathbf{c}, \mathbf{f}, \mathbf{i})$, and Compaction $(\mathbf{e}, \mathbf{g}, \mathbf{j})$ probes. The data were analyzed as in Figure 1 . The red dotted lines outline the corresponding histograms of the wild-type targeting complex. k-l, Quantification of the populations in low $(\square)$, median $(\square)$, and high $(\mathbf{\square})$ FRET states detected by the Proximal (k) or Distal (I) probes. m, Quantification of SR compaction, calculated from the fraction of targeting complex displaying high FRET $\left(\mathrm{E}^{*}=0.6-0.8\right)$ and subtracting the corresponding value in the histogram of ribosome-bound SR. All values are normalized to that of the wildtype targeting complex. Error bars in $\mathbf{k}-\mathbf{m}$ denote SD from at least three independent experiments. $\mathbf{n}$, BVA plot of SR compaction in the targeting complex. The black dashed curve depicts the static limit. Triangles denote the observed standard deviation for individual $\mathrm{E}^{*}$ bins $\left(S D_{E^{*}}\right)$ and were used to calculate the dynamic score (DS) and weighted dynamic score (WDS) (eqs 9-11 in Supplementary Methods). o, Rate constants for detachment of the NG-complex from the ribosome exit $\left(0.21 \pm 0.027 \mathrm{~s}^{-1}\right)$ and for distal site docking $\left(0.07 \pm 0.0007 \mathrm{~s}^{-1}\right)$ measured using the Proximal and Distal probes, respectively. Rate constants are from the data in Extended Data Fig. $6 \mathrm{~d}$ and are shown as mean $\pm \mathrm{SD}$, with $\mathrm{n}=3-5$. 

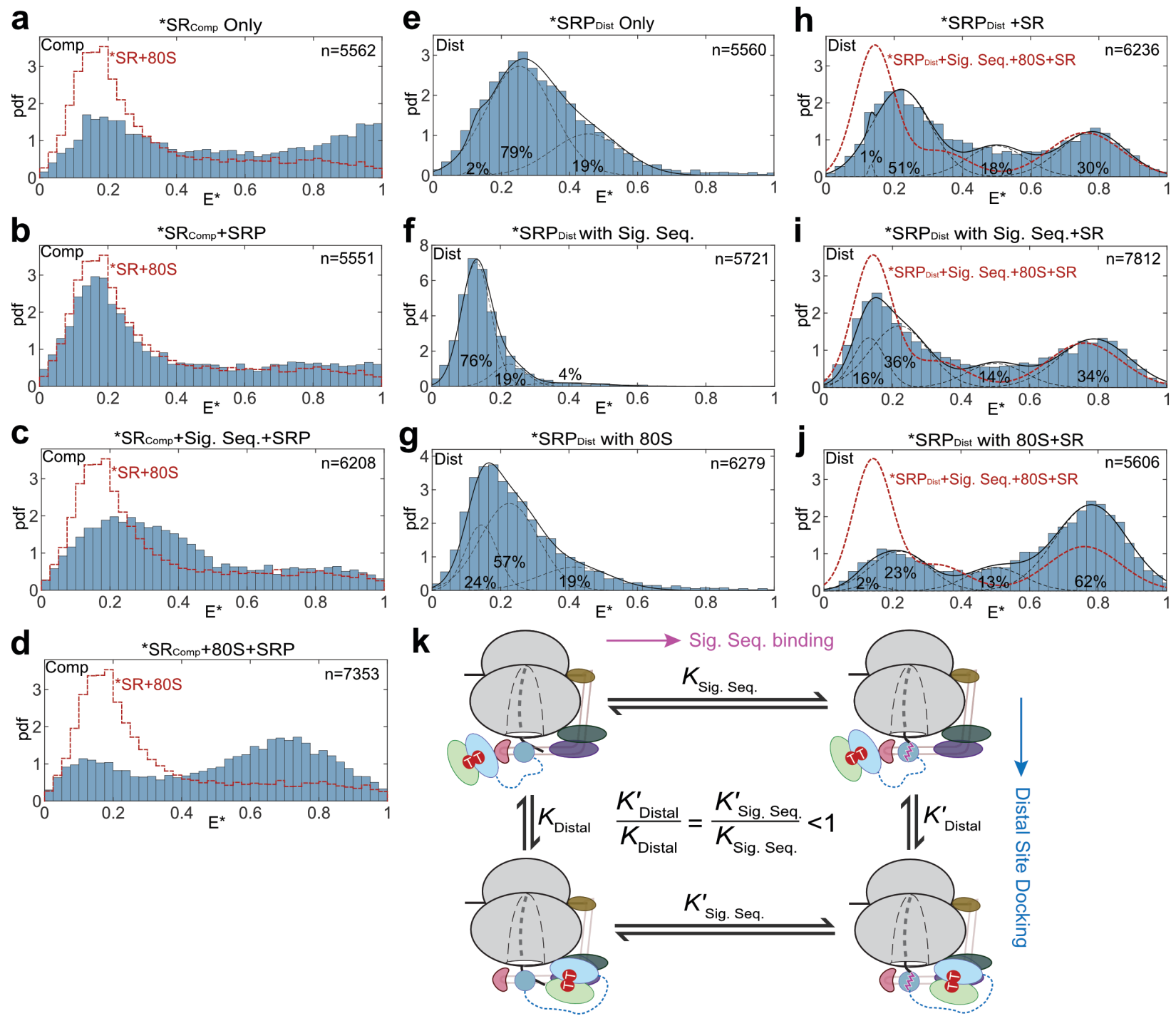

Figure 3. The ribosome and signal sequence regulate conformational changes in the targeting complex. a-d, smFRET histograms of SR Comp in the absence (a) or presence of SRP (b), signal sequence bound SRP (c), or ribosome-bound SRP (d). The red dotted lines outline the data for

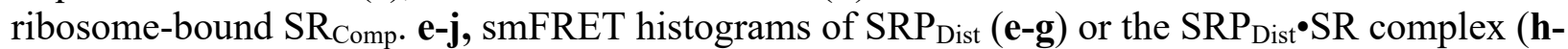
$\mathbf{j})$ in the absence $(\mathbf{e}, \mathbf{h})$ or presence of signal sequence $(\mathbf{f}, \mathbf{i})$ or ribosome $(\mathbf{g}, \mathbf{j})$. The red dotted lines in $\mathbf{h}$-j outline the data with the complete targeting complex. $\mathbf{k}$, Thermodynamic cycle analysis of the coupled equilibria of signal sequence binding and distal site docking of the NGcomplex. The less favorable distal site docking in the presence of signal sequence $\left(K^{\prime}{ }_{\text {Dist }}<K_{\text {Dist }}\right)$ implies weaker signal sequence binding in the distal state $\left(K_{\text {Sig. Seq. }}^{\prime}<K_{\text {Sig. Seq }}\right)$. 

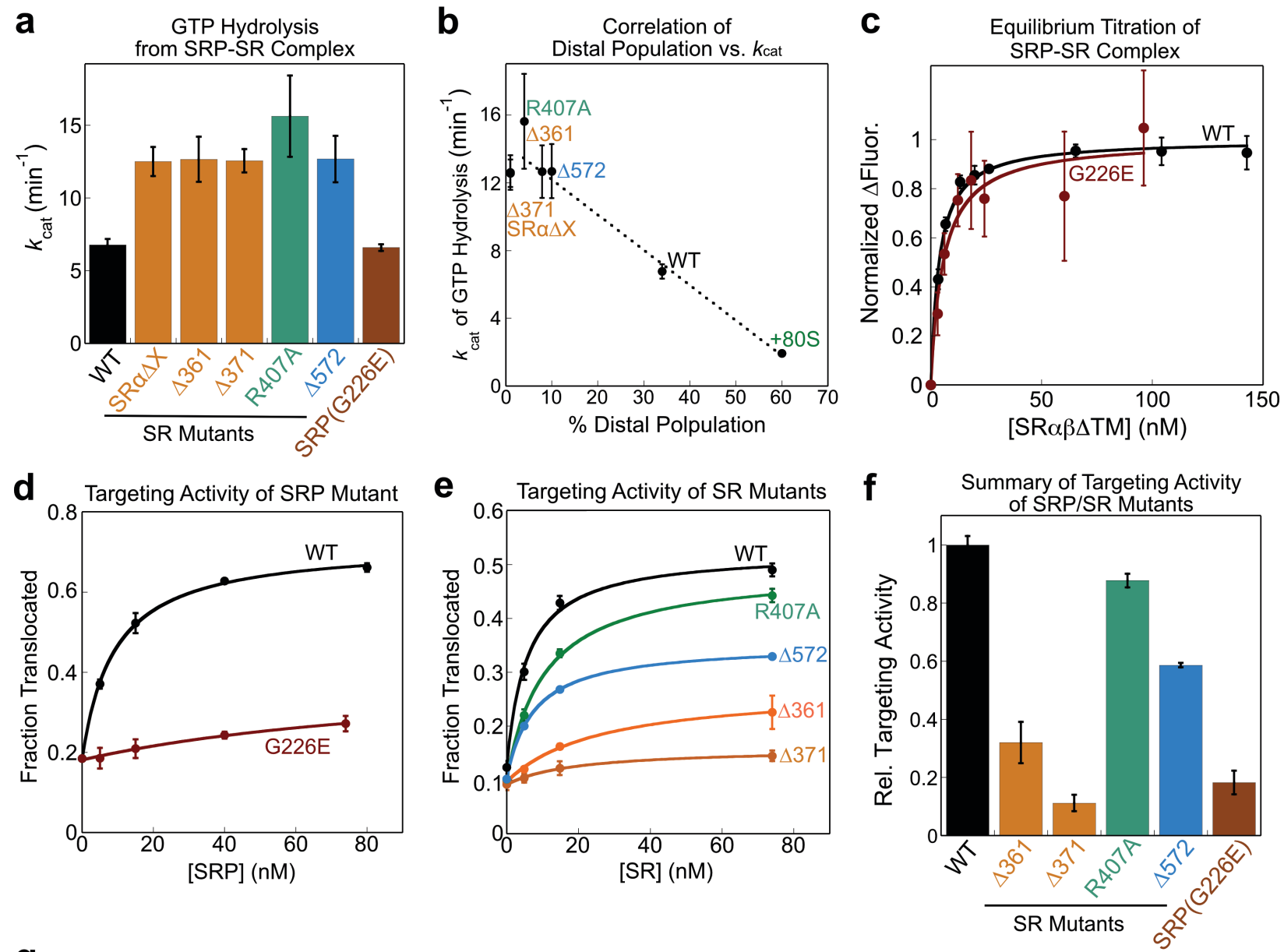

g

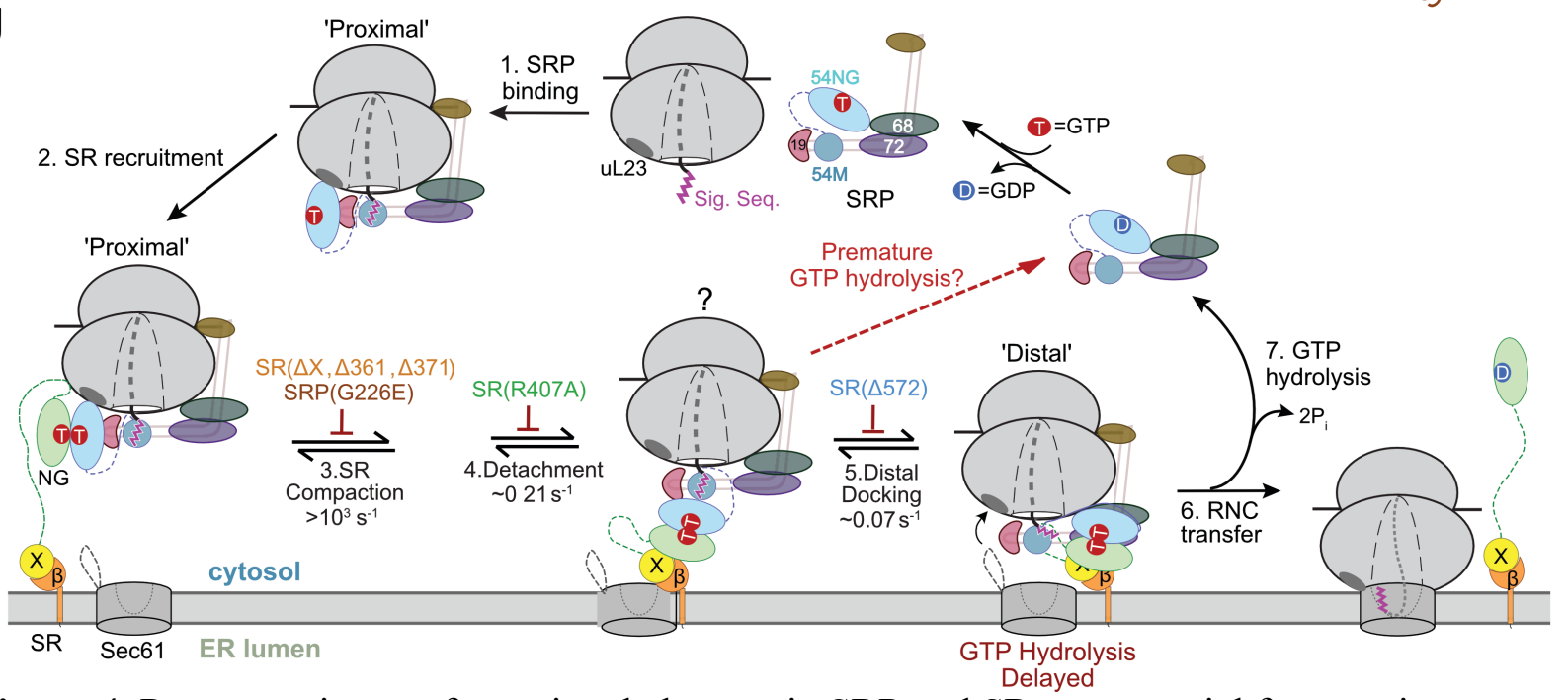

Figure 4. Post-targeting conformational changes in SRP and SR are essential for protein translocation. a, Summary of the rate constants of stimulated GTP hydrolysis $\left(k_{\mathrm{cat}}\right)$ in targeting complexes assembled with wildtype protein or the indicated SRP and SR mutants. Values are reported as mean $\pm \mathrm{SD}$, with $\mathrm{n}=3$. $\mathbf{b}, k_{\mathrm{cat}}$ negatively correlates with the fraction of SRP•SR complex in the distal conformation. The $k_{\text {cat }}$ for the reaction with ribosome-bound SRP•SR complex ('+80S') was measured previously ${ }^{5}$. c, Equilibrium titrations to measure the binding affinity between SRP(G226E) and SR. The data were fit to Eq 4 and gave equilibrium 
dissociation constants $\left(K_{\mathrm{d}}\right)$ of $3.7 \pm 0.35$ and $5.6 \pm 1.7 \mathrm{nM}$ for wildtype SRP and mutant $\mathrm{SRP}(\mathrm{G} 226 \mathrm{E})$. Error bars represent SD, with $\mathrm{n}=3$. d-e, Co-translational translocation of $\mathrm{pPL}$ mediated by wildtype or the indicated SRP (d) and SR (e) mutants. Translocation efficiencies were quantified from the data in Extended Data Fig. 8a, b and are reported as mean \pm SD, with $n$ $=2-3$. $\mathbf{f}$, Summary of the translocation efficiency of each mutant relative to the wildtype targeting complex at saturating protein concentrations. g, Model for co-translational protein targeting and translocation. SRP is pre-organized into the Proximal conformation upon binding to signal sequence bearing ribosomes (step 1) and recruits SR via interaction between their NGdomains (step 2). This induces SR compaction (step 3) to drive the detachment of the NGcomplex from the ribosome exit (step 4). The question mark on the targeting complex after step 4 denotes that the precise structure of this intermediate is unknown. Docking of the GTPase complex at the distal site (step 5) further primes the RNC for unloading to Sec61p (step 6). GTP hydrolysis, which dissociates SRP from SR (step 7), is delayed in the Distal state, and failure to reach the distal conformation could cause premature GTP hydrolysis that aborts targeting (dashed red arrows). 


\section{Methods}

Vectors. The vectors for expression of SRP and SR subunits and for fluorescence labeling of SRP19, SRP54 and SR $\alpha$ C-terminus have been described ${ }^{5}$. To fluorescently label SRP68, an Sfp recognition motif (ybbr6, DSLEFI) ${ }^{38}$ was inserted after Pro149 using Fastcloning. To fluorescently label SR $\beta \Delta \mathrm{TM}$, a longer Sfp recognition motif (ybbr11, DSLEFIASKLA) ${ }^{38}$ was inserted at the SR $\beta$ N-terminus using Fastcloning. Expression vectors for mutant SRP and SRs were generated using QuickChange mutagenesis protocol (Stratagene).

Biochemical Preparations. Wildtype and mutant SRP and SR proteins were expressed and purified as described ${ }^{5}$. Mammalian SRP was prepared as described ${ }^{5}$. Briefly, SRP protein subunits were expressed and purified in bacteria or yeast. A circularly permutated 7SL RNA variant was in vitro transcribed and purified on a denaturing polyacrylamide gel. SRP was assembled by first refolding 7SL RNA and sequentially adding SRP19, SRP68/72, SRP9/14, and SRP54. Holo-SRP was purified using a DEAE ion-exchange column. Unless otherwise specified, the C-terminus of human SRP54 was fused to the 4A10L signal sequence (hSRP54-4A10L) to generate signal sequence-bound SRP, as described ${ }^{5}$. Ribosome from rabbit reticulocyte lysate (RRL) was purified by ultracentrifugation through a sucrose gradient, as described ${ }^{5}$. The use of ribosome and signal sequence fusion to SRP54 reproduced the effects of signal sequence-bearing RNCs on the conformation and activity of $\mathrm{SRP}^{5}$.

Fluorescence Labeling. SRP54(C12), SRP54(C47), and SRP19(C64) were labeled with Atto550, Atto647N, or Cy3B using maleimide chemistry as described ${ }^{5}$. 
SRP68/72 was labeled via Sfp-mediated conjugation of CoA-Atto647N or CoA-Cy3B at Ser2 in ybbr6 tagged SRP68 following the procedure described in ${ }^{38}$. The labeling reaction contained 0.4 molar ratio of protein to Sfp enzyme and a 3-fold excess of CoA-Atto647N (or CoA-Cy3B), and was carried out for 20 minutes at room temperature in Sfp-labeling Buffer (50 mM KHEPES (7.5), $10 \mathrm{mM} \mathrm{MgCl} 2,150 \mathrm{mM} \mathrm{NaCl}$, and 20\% glycerol). Labeling efficiency was close to $100 \%$. Labeled SRP68/72 was immediately used for SRP assembly.

$\mathrm{SR} \alpha \beta \Delta \mathrm{TM}$ was doubly labeled via sortase-mediated ligation at the $\mathrm{SR} \alpha \mathrm{C}$-terminus and Sfp-mediated conjugation of CoA-dye at the N-terminal ybbr11 tag on SR $\beta$. The labeling reaction contained 0.4 molar ratio of Sfp to protein and a 2-fold excess of CoA-Atto647N (or CoA-Atto550), and was carried out for 30 minutes at room temperature in Sfp-labeling Buffer. A 4-fold molar excess of sortase,10-fold excess of GGGC-Atto550 (or GGGC-Atto647N), and 0.1 volume of 10X Sortase Buffer (500mM Tris- $\mathrm{HCl} 7.5,1.5 \mathrm{M} \mathrm{NaCl}$, and $\left.100 \mathrm{mM} \mathrm{CaCl}_{2}\right)$ was then added, and the labeling reaction was carried out for an additional 3 hours at room temperature. Labeled SRP68/72 was purified using Ni-Sepharose resin. Labeling efficiency was close to $100 \%$ for the Sfp reaction and $\sim 60-70 \%$ for the sortase reaction.

Biochemical Assays. All proteins except for SRP were ultracentrifuged at $4{ }^{\circ} \mathrm{C}, 100,000 \mathrm{rpm}$ in a TLA100 rotor for 30 - 60 minutes to remove aggregates before all assays. GTPase reactions were performed in SRP Assay Buffer (50 mM KHEPES (pH 7.5), 150 mM KOAc, 5 mM $\operatorname{Mg}(\mathrm{OAc})_{2}, 10 \%$ glycerol, $2 \mathrm{mM}$ DTT, and $0.04 \%$ Nikkol $)$ at $25{ }^{\circ} \mathrm{C}$ and were followed and analyzed as described ${ }^{5,39}$. Details for the determination of the GTPase rate constants are described in Supplementary Methods. Co-translational targeting and translocation of pPL into salt-washed and trypsin digested rough ER microsomes (TKRM) were performed and analyzed 
as described in Supplementary Methods. Steady-state fluorescence measurements were carried out on a Fluorolog 3-22 spectrofluorometer (Jobin Yvon) at $25{ }^{\circ} \mathrm{C}$ in SRP Assay Buffer. Acquisition and analyses of fluorescence data are described in Supplementary Methods.

smFRET . Measurements were performed as described ${ }^{5,25,26}$. Labeled SRP was diluted to 100-200 pM in SRP Assay Buffer containing $200 \mu \mathrm{M}$ non-hydrolysable GTP (GppNHp), 150 nM 80S, and 1.5 $\mu \mathrm{M} \operatorname{SR} \alpha \beta \Delta \mathrm{TM}$ where indicated. To measure the conformation of SR, doubly labeled $\mathrm{SR} \alpha \beta \Delta \mathrm{TM}$ was diluted to $100-200 \mathrm{pM}$ in SRP Assay Buffer containing $200 \mu \mathrm{M}$ GppNHp, 400 $\mathrm{nM} 80 \mathrm{~S}$, and $400 \mathrm{nM}$ SRP or SRP-4A10L where indicated. Data were collected over 30-60 min using an ALEX-FAMS setup with two single-photon Avalanche photodiodes (Perkin Elmer) and $532 \mathrm{~nm}$ (CNI laser) and $638 \mathrm{~nm}$ (Opto Engine LLC) continuous wave lasers operating at $150 \mu \mathrm{W}$ and $70 \mu \mathrm{W}$, respectively. Analysis of $\mu \mathrm{s}-\mathrm{ALEX}$ data are described in Supplementary Methods.

ACKNOWLEDGMENT. We thank S. Chandrasekar and H. Hsieh for sharing reagents, A. Jomaa and N. Ban for helpful discussions, and members of the Shan lab for comments on the manuscript. This work was supported by National Institutes of Health grant GM078024 and the Gordon and Betty Moore Foundation through grant GBMF2939 to S.-o. Shan, and by National Institutes of Health grant GM130942 and Dean Willard Chair funds to S.W.

AUTHOR CONTRIBUTIONS. J.L., Y.H., and S.S. designed research; J.L., Y.H., R.Q, and X.S. performed biochemical experiments and analyzed data; J.L., R.Q., and S.C. performed $\mu$ s-ALEX experiments and analyzed data; S.W. provided guidance for $\mu$ s-ALEX analysis; J.L. and S.S. wrote the manuscript with input from S.C. and S.W. 


\section{References}

1. Akopian, D., Shen, K., Zhang, X. \& Shan, S. Signal Recognition Particle: An Essential Protein-Targeting Machine. Annu. Rev. Biochem. 82, 693-721 (2013).

2. Zhang, X. \& Shan, S. Fidelity of Cotranslational Protein Targeting by the Signal Recognition Particle. Annu. Rev. Biophys. 43, 381-408 (2014).

3. Walter, P. \& Blobel, G. [53] Signal recognition particle: A ribonucleoprotein required for cotranslational translocation of proteins, isolation and properties. Methods in Enzymology 96, 682-691 (1983).

4. Mandon, E. C., Jiang, Y. \& Gilmore, R. Dual recognition of the ribosome and the signal recognition particle by the SRP receptor during protein targeting to the endoplasmic reticulum. J. Cell Biol. 162, 575-585 (2003).

5. Lee, J. H. et al. Sequential activation of human signal recognition particle by the ribosome and signal sequence drives efficient protein targeting. Proc. Natl. Acad. Sci. 115, E5487E5496 (2018).

6. Walter, P. \& Johnson, A. E. Signal Sequence Recognition and Protein Targeting to the Endoplasmic Reticulum Membrane. Annu. Rev. Cell Biol. 10, 87-119 (1994).

7. Carapito, R. et al. Mutations in signal recognition particle SRP54 cause syndromic neutropenia with Shwachman-Diamond-like features. J. Clin. Invest. 127, 4090-4103 (2017).

8. Bellanné-Chantelot, C. et al. Mutations in the SRP54 gene cause severe congenital neutropenia as well as Shwachman-Diamond - Like syndrome. Blood 132, 1318-1331 (2018).

9. Voorhees, R. M. \& Hegde, R. S. Structures of the scanning and engaged states of the 
mammalian SRP-ribosome complex. eLife 4, e07975 (2015).

10. Flanagan, J. J. et al. Signal Recognition Particle Binds to Ribosome-bound Signal

Sequences with Fluorescence-detected Subnanomolar Affinity That Does Not Diminish as the Nascent Chain Lengthens. J. Biol. Chem. 278, 18628-18637 (2003).

11. Halic, M. et al. Structure of the signal recognition particle interacting with the elongationarrested ribosome. Nature 427, 808-814 (2004).

12. Voorhees, R. M., Fernández, I. S., Scheres, S. H. W. \& Hegde, R. S. Structure of the Mammalian Ribosome-Sec61 Complex to 3.4 Å Resolution. Cell 157, 1632-1643 (2014).

13. Voorhees, R. M. \& Hegde, R. S. Toward a structural understanding of co-translational protein translocation. Curr. Opin. Cell Biol. 41, 91-99 (2016).

14. Tajima, S., Lauffer, L., Rath, V. L. \& Walter, P. The signal recognition particle receptor is a complex that contains two distinct polypeptide chains. J. Cell Biol. 103, 1167-1178 (1986).

15. Schwartz, T. \& Blobel, G. Structural basis for the function of the $\beta$ subunit of the eukaryotic signal recognition particle receptor. Cell 112, 793-803 (2003).

16. Jadhav, B. et al. Mammalian SRP receptor switches the Sec61 translocase from Sec62 to SRP-dependent translocation. Nat. Commun. 6, 10133 (2015).

17. Hwang Fu, Y.-H., Chandrasekar, S., Lee, J. H. \& Shan, S. A molecular recognition feature mediates ribosome-induced SRP-receptor assembly during protein targeting. J. Cell Biol. 218, 3307-3319 (2019).

18. Connolly, T., Rapiejko, P. \& Gilmore, R. Requirement of GTP hydrolysis for dissociation of the signal recognition particle from its receptor. Science 252, 1171-1173 (1991).

19. Rapiejko, P. J. \& Gilmore, R. Empty site forms of the SRP54 and SR $\alpha$ GTPase mediate 
targeting of ribosome-nascent chain complexes to the endoplasmic reticulum. Cell $\mathbf{8 9}$, $703-713$ (1997).

20. Wilson, C., Connolly, T., Morrison, T. \& Gilmore, R. Integration of membrane proteins into the endoplasmic reticulum requires GTP. J. Cell Biol. 107, 69-77 (1988).

21. Chitwood, P. J., Juszkiewicz, S., Guna, A., Shao, S. \& Hegde, R. S. EMC Is Required to Initiate Accurate Membrane Protein Topogenesis. Cell 175, 1507-1519.e16 (2018).

22. Itzhak, D. N. et al. The ER membrane protein complex interacts cotranslationally to enable biogenesis of multipass membrane proteins. eLife 7, 1-23 (2018).

23. Halic, M. Signal Recognition Particle Receptor Exposes the Ribosomal Translocon Binding Site. Science 312, 745-747 (2006).

24. Kobayashi, K. et al. Structure of a prehandover mammalian ribosomal SRP·SRP receptor targeting complex. Science 360, 323-327 (2018).

25. Kapanidis, A. N. et al. Fluorescence-aided molecule sorting: Analysis of structure and interactions by alternating-laser excitation of single molecules. Proc. Natl. Acad. Sci. 101, 8936-8941 (2004).

26. Kapanidis, A. N. et al. Alternating-Laser Excitation of Single Molecules. Acc. Chem. Res. 38, 523-533 (2005).

27. Ingargiola, A., Lerner, E., Chung, S., Weiss, S. \& Michalet, X. FRETBursts: An Open Source Toolkit for Analysis of Freely-Diffusing Single-Molecule FRET. PLoS One 11, e0160716 (2016)

28. Ogg, S. C., Barz, W. P. \& Walter, P. A Functional GTPase Domain, but not its Transmembrane Domain, is Required for Function of the SRP Receptor $\beta$-subunit. J. Cell Biol. 142, 341-354 (1998). 
29. Torella, J. P., Holden, S. J., Santoso, Y., Hohlbein, J. \& Kapanidis, A. N. Identifying Molecular Dynamics in Single-Molecule FRET Experiments with Burst Variance Analysis. Biophys. J. 100, 1568-1577 (2011).

30. Robb, N. C. et al. The transcription bubble of the RNA polymerase-promoter open complex exhibits conformational heterogeneity and millisecond-scale dynamics: Implications for transcription start-site selection. J. Mol. Biol. 425, 875-885 (2013).

31. Chio, U. S., Chung, S., Weiss, S. \& Shan, S. A protean clamp guides membrane targeting of tail-anchored proteins. Proc. Natl. Acad. Sci. 114, E8585-E8594 (2017).

32. Shan, S., Stroud, R. M. \& Walter, P. Mechanism of Association and Reciprocal Activation of Two GTPases. PLoS Biol. 2, e320 (2004).

33. Egea, P. F. et al. Substrate twinning activates the signal recognition particle and its receptor. Nature 427, 215-221 (2004).

34. Zhang, X., Kung, S. \& Shan, S. Demonstration of a Multistep Mechanism for Assembly of the SRP.SRP Receptor Complex: Implications for the Catalytic Role of SRP RNA. $J$. Mol. Biol. 381, 581-593 (2008).

35. Shen, K. et al. Molecular Mechanism of GTPase Activation at the Signal Recognition Particle (SRP) RNA Distal End. J. Biol. Chem. 288, 36385-36397 (2013).

36. Voigts-Hoffmann, F. et al. The Structural Basis of FtsY Recruitment and GTPase Activation by SRP RNA. Mol. Cell 52, 643-654 (2013).

37. Shan, S., Chandrasekar, S. \& Walter, P. Conformational changes in the GTPase modules of the signal reception particle and its receptor drive initiation of protein translocation. $J$. Cell Biol. 178, 611-620 (2007).

38. Yin, J., Lin, A. J., Golan, D. E. \& Walsh, C. T. Site-specific protein labeling by Sfp 

available under aCC-BY-NC-ND 4.0 International license.

phosphopantetheinyl transferase. Nat. Protoc. 1, 280-285 (2006).

39. Peluso, P., Shan, S., Nock, S., Herschlag, D. \& Walter, P. Role of SRP RNA in the GTPase Cycles of Ffh and FtsY †. Biochemistry 40, 15224-15233 (2001). 